\title{
ETHICAL STANDARDS IN ROBOTICS AND AI: WHAT ARE THEY AND WHY THEY MATTER
}

\author{
ALAN WINFIELD \\ University of West England, UK
}

In response to concerns over the ethical and societal impact of robotics and AI a new generation of ethical standards are emerging. In this talk I will introduce these standards, including both British Standard BS8611 Guide to the ethical design of robots and robotic systems and the IEEE P700X series of 'human standards' currently in development. As a case study I will focus on one of these emerging standards: P7001 on Transparency of Autonomous Systems. I will set these ethical standards within the wider context of Ethical Governance, and ask the question: what is good ethical governance in robotics? Standards, like open science, are a trust technology. Without ethical standards, it is hard to see how robots and AIs will be trusted and widely accepted, and without that acceptance their great promise will not be realized. 